Torsten Wilholt

\title{
Die Objektivität der Wissenschaften als soziales Phänomen
}

\begin{abstract}
Scientific procedures are widely expected to be unbiased, in the sense that they do not single out one specific set of claims about which they yield false results more often than about others. This assumed feature of the practices of science can be called procedural objectivity. I argue that attempts to analyze procedural objectivity on the level of individual rationality fail. The appropriate balance of inductive risks for each scientific investigation hinges upon value judgments for which no binding, ,neutral' standard can be derived from universal principles. I make the case that the perspective of social epistemology offers a much more promising approach to establish a substantial conception of procedural objectivity. I examine two genuinely social elements of the sciences' procedural objectivity. One consists in conventional standards, which are adopted by research communities in order to facilitate epistemic trust and which impose constraints on methodological choices that affect the balance of inductive risks. The other is constituted by the plurality of approaches within research communities and the mechanism of mutual criticism. Procedural objectivity in science thus becomes understandable as a social phenomenon.
\end{abstract}

\section{Einleitung}

Dass Wissenschaft ein Kollektivunternehmen ist, ist eine beinahe banale Feststellung. Eine arbeitsteilige Organisationsweise wissenschaftlicher Forschung ist angesichts des schieren Umfangs der insgesamt anfallenden Arbeit praktisch alternativlos. Dieser Befund gilt offensichtlich auch für die kognitive Arbeit der Wissenschaftler. Weniger offensichtlich ist, wie tiefgreifend und wesentlich dieser soziale Charakter wissenschaftlicher Forschung ist. Für lange Zeit herrschte in der Wissenschaftstheorie die Auffassung vor, dass für wissenschaftliche Forschung bestimmte Methoden der Erkenntnisgewinnung kennzeichnend seien, welche im Prinzip von jedem beliebigen einzelnen Individuum zur Anwendung gebracht werden könnten und deren spezifische Charakteristika für den großen epistemischen Erfolg der Wissenschaften verantwortlich seien. Die besonders seit Thomas Kuhn (1962) ausführlich betriebene Kritik an diesem individualistischen Wissenschaftsverständnis, in deren Folge der häufige Einfluss von Autoritätsverhältnissen, Aushandlungsprozessen und vielen weiteren wesentlich sozialen Vorgängen auf Ergebnisse der Forschung nachgewiesen werden konnte, ging oft 
mit einer tiefen Skepsis gegenüber den Erkenntnisansprüchen der Wissenschaften einher (vgl. z.B. Latour/Woolgar 1979; Collins 1985).

Im Gegensatz zu diesen beiden Extrempositionen wird in der gegenwärtigen sozialen Erkenntnistheorie häufig die konstruktive Auffassung vertreten, dass der epistemische Erfolg der Wissenschaften ein wesentliches Ergebnis auch ihrer sozialen Organisationsform ist. ${ }^{1}$ Die Vorgehensweisen der Wissenschaften sind demnach in wesentlicher Weise sozial. Im Folgenden stelle ich einige Überlegungen vor, die diese Sichtweise meines Erachtens in besonders nachdrücklicher Weise stützen. Insbesondere werde ich dafürhalten, dass eine überzeugende Analyse der Objektivität der Wissenschaften nur möglich wird, wenn man sie als soziales Phänomen betrachtet.

Von wissenschaftlicher Objektivität kann in verschiedenen Sinnen die Rede sein (vgl. Douglas 2004). In der hitzig geführten Kontroverse zwischen wissenschaftlichen Realisten und sozialen Konstruktivisten (s. dazu Hacking 1999) ging (und geht) es um die Frage, ob die Wissenschaften auf objektive Wahrheiten abzielen, wobei darunter treue Repräsentationen einer Realität zu verstehen sind, welche unabhängig von den sie erforschenden Subjekten ist. Dies ist nicht (primär) der Sinn von ,Objektivität', der in der vorliegenden Abhandlung im Vordergrund stehen soll. Vielmehr werde ich mich auf denjenigen Sinn konzentrieren, den man (Megill 1994 folgend) als prozedurale Objektivität bezeichnen könnte, denn er bezieht sich nicht auf den Weltbezug einzelner Theorien und Aussagen, sondern auf Methoden und Vorgänge der Erkenntnisgewinnung. Wenn wir ein epistemisches Verfahren objektiv nennen, meinen wir damit im Kern, dass es frei von Einseitigkeiten sei - das heißt, grob gesagt, dass es nicht bei gewissen inhaltlich bestimmten Arten von Behauptungen, zu deren Überprüfung es grundsätzlich geeignet ist, systematisch häufiger falsche Ergebnisse zeitige als bei anderen. Von den Verfahren der Wissenschaften erwarten wir im Allgemeinen eine ausgeprägte prozedurale Objektivität. Wir erwarten, dass sie möglichst frei von individuell verursachten Einseitigkeiten sind, und dass die wissenschaftlichen Methoden deshalb dieselben Ergebnisse hervorzubringen tendieren, gleich welches Individuum oder welche Gruppe von Individuen sie anwendet. Ebenso erwarten wir, dass sie auch möglichst frei von kollektiv geprägten Einseitigkeiten sind, welche etwa die gemeinsamen Vorurteile der Gesellschaft, innerhalb derer wissenschaftliche Erkenntnisgewinnung stattfindet, widerspiegeln. Zwar hat die historische und sozialwissenschaftliche Wissenschaftsforschung gezeigt, dass die real existierende wissenschaftliche Wissensgewinnung immer wieder weit vom Ideal prozeduraler Objektivität entfernt ist. Aber das hindert uns nicht daran, die Erwartung prozeduraler Objektivität und das beständige und systematische Bemühen um sie als entscheidende Merkmale wissenschaftlicher Wissensgewinnung zu identifizieren. Die Frage nach der objektiven Wahrheit im Sinne der Realismusdebatte betrifft wissenschaftliche und außerwissenschaftliche Sätze gleichermaßen. Dagegen ist prozedurale Objektivität der Verfahren etwas, durch das sich die Methoden gerade der wissenschaftlichen Erkenntnisgewinnung in besonderer Weise auszeichnen sollen. $\mathrm{Ob}$ und gegebenenfalls wo sie in

1 Aus verschiedenen Blickwinkeln haben diese Sichtweise in jüngerer Zeit Philip Kitcher (1993, 2001), Helen Longino (1990, 2002) und Alvin Goldman (1999, Kap. 8) vertreten. 
den Wissenschaften zu finden sei, ist oft eine brisante Frage, die auch außerhalb der akademischen Wissenschaftsreflexion vielfach mit Ernst gestellt wird. Den Focus auf die prozedurale Objektivität zu richten, verspricht daher auch, der Diskussion eine größere Praxisrelevanz zu verleihen.

\section{Prozedurale Objektivität und induktive Risiken}

Um den in Rede stehenden Sinn von Objektivität genauer zu analysieren, bietet es sich an, einen Fall zu betrachten, in dem wir intuitiv von einem Versagen prozeduraler Objektivität sprechen würden. Betrachten wir das folgende Beispiel: Bisphenol A ist eine bei der Herstellung von Kunststoffen verwendete Substanz. Dass sie grundsätzlich toxisch ist und dass dies mit ihrer Ähnlichkeit zu menschlichem Östrogen zusammenhängt, ist nicht umstritten. Die Frage jedoch, inwieweit auch von geringen Dosen, wie sie etwa aus Kunststoffabdichtungen von Lebensmittelkonserven austreten können, eine Gesundheitsgefährdung ausgeht, führte zu einer wissenschaftlichen und teilweise auch öffentlich in den Medien ausgetragenen Kontroverse. Zu ihrer Klärung wurde eine ganze Reihe von Tierversuchen durchgeführt. 2005 stellten die Biomediziner Frederick vom Saal und Claude Hughes fest, dass 90\% der von öffentlichen Auftraggebern veranlassten Studien eine signifikante Wirkung geringer Dosen konstatierten, während das keine einzige der zahlreichen von der Industrie veranlassten Studien tat (vom Saal/Hughes 2005). Es stellte sich heraus, dass viele der Industriestudien auf einen ganz bestimmten Stamm von Laborratten zurückgegriffen hatten, deren Organismen für ihre nicht vollständige, aber doch weitgehende Unempfindlichkeit gegen östrogene Substanzen bekannt sind (ebd.; vom Saal/Welshons 2005). Offenbar hat diese Wahl des Versuchstiers die Wahrscheinlichkeit eines negativen Ergebnisses der entsprechenden Studien erhöht.

Genau in dieser Verschiebung der Fehlerwahrscheinlichkeit könnte man eine Möglichkeit sehen, prozedurale Objektivität - beziehungsweise, wie in diesem Fall, den Mangel daran - auf der Ebene individueller methodologischer Entscheidungen zu analysieren. Um dies näher zu erläutern, bietet es sich an, den von Carl Hempel (1965, 91-2) geprägten Begriff des induktiven Risikos zu bemühen. In jeder wissenschaftlichen Untersuchung, bei der eine Hypothese überprüft werden soll, gibt es zwei verschiedene Arten von induktiven Risiken: Das Risiko, dass die Untersuchung zur Akzeptanz der Hypothese führen könnte, obwohl diese tatsächlich falsch ist, und das Risiko, dass sie zur Zurückweisung der Hypothese führen könnte, obwohl diese in Wirklichkeit zutrifft. Insgesamt reduzieren lassen sich diese induktiven Risiken immer nur durch größeren empirischen Aufwand (im Allgemeinen durch Vergrößerung der Datenbasis). Aber auch bei gleichbleibendem Aufwand ist immer ein Trade-Off zwischen beiden Arten möglich, bei denen also eines der beiden Risiken verringert wird, während das andere zunimmt. Dies lässt sich durch ein verändertes experimentelles Design oder eine geänderte Interpretation der Daten erreichen. So wurde in den erwähnten Industriestudien zu Bisphenol A durch die Wahl des Versuchstiers 
das Risiko eines falsch-positiven Ergebnisses reduziert, zugleich aber das Risiko eines falsch-negativen Ergebnisses erhöht.

In genau diesem Sinne könnte man versuchen, das Ideal der prozeduralen Objektivität wie folgt zu charakterisieren. Prozedural objektiv wäre ein solches Vorgehen, das auf jeden solchen Trade-Off verzichtet - ein Vorgehen, bei dem keine einseitigen Verschiebungen an der Balance der induktiven Risiken vorgenommen werden. Dies wäre ein Verständnis prozeduraler Objektivität, das sich auf der Ebene individueller methodologischer Entscheidungen in Anschlag bringen ließe. Auf diese Weise könnte man versuchen, prozedurale Objektivität etwa als Unvoreingenommenheit gegenüber den möglichen Ergebnissen der Untersuchung aufzufassen.

Offenbar beruht diese Auffassung von Objektivität als Unvoreingenommenheit aber auf einer kritischen Voraussetzung, nämlich auf der Annahme, dass es für jede Untersuchung ein bestimmtes ,richtiges ${ }^{6}$ oder ,neutrales ${ }^{6}$ Verhältnis der induktiven Risiken gebe. Eben diese Voraussetzung erweist sich bei näherem Hinsehen als unhaltbar. Um dies genau zu sehen, müssen wir ein wenig ausholen.

Es ist eine seit langem bekannte Eigentümlichkeit induktiver Erkenntnisprozesse, dass Statistik und induktive Logik uns keine eindeutigen Vorgaben darüber machen, wie sicher wir sein müssen, bevor wir eine Hypothese akzeptieren - oder darüber, wie sicher wir sein müssen, bevor wir sie zurückweisen (vgl. z.B. Churchman 1948, Kap. 15). Die Entscheidung über diese beiden Fragen wäre aber genau die Entscheidung über das ,richtige' Verhältnis induktiver Risiken. Sie erweist sich selbst als eine jeweils von Fall zu Fall zu treffende methodologische Entscheidung, die nur davon abhängig sein kann, für wie schwerwiegend wir den jeweiligen möglichen Fehler und für wie wertvoll wir das entsprechende richtige Ergebnis halten würden (Rudner 1953). Diese Fragen scheinen aber im Bereich individueller Wertbeurteilungen zu liegen und keine verallgemeinerbaren ,richtigen' Antworten zu besitzen. ${ }^{2}$

Nun könnte man sich möglicherweise auf den Standpunkt stellen, dass gerade diese Fragen vor dem Hintergrund des Ideals der Unvoreingenommenheit doch eindeutig bestimmte Antworten besäßen: Dem unvoreingenommenen Wissenschaftler dürfe es nämlich nur um die Wahrheit gehen. Deshalb müsse ihm jedes korrekte Ergebnis gleich willkommen und jedes falsche gleich unerwünscht sein. Infolgedessen müssten aus der Sicht des unvoreingenommenen Wissenschaftlers falsch-positive und falsch-negative Fehler immer genau gleich schwerwiegend erscheinen und daher nur solche Verteilungen der induktiven Risiken akzeptabel sein, bei der beide Fehlerwahrscheinlichkeiten genau gleich zu veranschlagen sind.

Diese Vorstellung, die bedeuten würde, dass in jeder wissenschaftlichen Untersuchung die zahlreichen methodologischen Entscheidungen über das Design der Studie oder des Experiments und über die Interpretation und Auswertung der Daten, die alle einen Einfluss auf die Proportionen der induktiven Risiken haben, genau so aufeinander abgestimmt werden müssen, dass sich die induktiven Risiken stets genau im Gleichgewicht befinden, ist meilenweit von tatsächlichen und bewährten epistemischen Praktiken der Wissenschaften entfernt. Das allein

${ }^{2}$ Diese bereits bei Churchman und Rudner angelegte Argumentation hat in jüngerer Zeit durch Douglas 2000 eine Wiederbelebung erfahren. 
wäre möglicherweise noch kein allzu schwerwiegender Einwand, denn schließlich geht es hier um die Analyse eines normativen Ideals. Es zeigt sich aber, dass die Gleichgewichtskonzeption sich sogar unmöglich mit wesentlichen Merkmalen der wissenschaftlichen Wahrheitssuche vereinbaren lässt. Denn diese wäre ein banales und geradezu groteskes Unterfangen, wenn es ihr darum ginge, jede Wahrheit mit derselben Intensität zu verfolgen, ganz gleich, ob es nun um die Existenz des Higgs-Bosons geht oder darum, wieviele Gänseblümchen am 14. Juli 2009 auf Bornholm geblüht haben. Die Wissenschaften suchen nicht einfach Wahrheiten, sondern signifikante Wahrheiten (Kitcher 1993, Kap. 4; 2001, Kap. 6). Der relevante Begriff von Signifikanz wird dabei sowohl von der Bedeutung einer neuen Wahrheit für die systematische Organisation der vorhandenen Wissensbestände bestimmt, als auch von ihrer potentiellen Bedeutung für die Lösung praktischer Probleme. Keineswegs ist zu erwarten, dass eine positive und eine negative Antwort auf dieselbe Frage typischerweise gleich signifikant wären: Die Erkenntnis, dass es sich bei den von Stanley Pons und Martin Fleischmann experimentell erzeugten Vorgängen nicht um kalte Fusion handelte, besaß nicht dieselbe Signifikanz, die die entgegengesetzte Behauptung besessen hätte, wenn sie sich als wahr erwiesen hätte. Wenn nicht alle Wahrheiten gleich bedeutsam und nicht alle Irrtümer gleich bedrohlich sind, kann aber auch ein striktes Gleichgewicht der induktiven Risiken nicht in jedem Fall die methodologische Richtschnur abgeben.

Diese Einsicht hat eine Entsprechung in der heute in der Wissenschaftsphilosophie weithin anerkannten Feststellung, dass es für das Verständnis wissenschaftlicher Praktiken nicht ausreicht, als einzigen von ihr anerkannten Wert das bloße Verfolgen von empirisch überprüfbarer Wahrheit anzusehen. Es muss vielmehr unterstellt werden, dass sie darüber hinaus noch auf eine ganze Reihe von Werten abzielen: Einfachheit, Vereinheitlichungs- und Erklärungskraft sowie die Fruchtbarkeit für den weiteren Fortgang des Forschungsprogramms sind Beispiele für solche ,epistemischen Werte' (Kuhn 1977; McMullin 1983). Der potentielle Gesamtwert einer untersuchten Hypothese hinsichtlich aller epistemischen Werte, sollte sie sich als wahr herausstellen, stimmt im Allgemeinen nicht mit dem Gesamtwert ihrer Verneinung (im Fall, dass diese zutrifft) überein. Eine strikte Gleichverteilung der induktiven Risiken würde diesem Umstand nicht Rechnung tragen. Auf diese Weise lässt sich Objektivität also nicht definieren.

Als einen letzten Versuch, doch noch für jede Untersuchung einen Standard für die ,richtige' Verteilung induktiver Risiken zu finden, um so die prozedurale Objektivität allein auf der Ebene individueller methodologischer Entscheidungen beschreiben zu können, möchte ich die Möglichkeit einer objektiv gegebenen epistemischen Nutzenfunktion in Betracht ziehen. Dies würde auf der Annahme aufbauen, dass sich eine strikte Trennung zwischen epistemischen und nichtepistemischen Werten durchhalten lässt. Wäre das möglich, dann könnte man mutmaßen, dass sich auf Grundlage allein der epistemischen Werte jedem möglichen Ausgang einer wissenschaftlichen Untersuchung ein bestimmter, objektiv richtiger Gesamtwert zuweisen ließe. Diese Wertungen würden sozusagen eine ,rein epistemische' Nutzenfunktion für die verschiedenen möglichen Ausgänge der Untersuchung festlegen, auf deren Grundlage sich die richtige Verteilung 
der akzeptablen induktiven Risiken entscheidungstheoretisch bestimmen ließe. ${ }^{3}$ Doch eine genaue Abgrenzung der ,zulässigen“ Werte, die einen Einfluss auf die rein epistemische Nutzenfunktion haben dürfen, erweist sich als schwierig. Der klassischen Definition epistemischer Werte (McMullin 1983) zufolge handelt es sich dabei um diejenigen Charakteristika einer Hypothese, die wir für Anzeichen ihrer Wahrheit halten. Dies kann jedoch für keines der genannten Charakteristika wie Einfachheit, Vereinheitlichungskraft und dergleichen als gut bestätigte und allgemein verbindliche Erkenntnis gelten. So wird ,unsere“ Beantwortung der Frage, ob es sich bei ihnen um Wahrheitsindikatoren handelt, häufig Gegenstand von Kontroversen sein, bei denen vernünftig argumentierende Akteure begründet zu verschiedenen Auffassungen gelangen können (vgl. Kourany 2003, 9). Beispielsweise haben feministische Wissenschaftstheoretikerinnen das weithin als epistemischer Wert anerkannte Ideal der ,externen Konsistenz' (neuer Theorien mit älteren, etablierten) dahingehend kritisiert, dass es eher ein Indikator der Zufriedenheit seiner Anhänger mit dem Status Quo als ein Wahrheitsindikator sei (Longino 1996, 51-2).

Selbst wenn es eine unkontroverse, bestimmte Menge epistemischer Werte gäbe, würde dies nicht gewährleisten, dass sich aus ihrem Zusammenwirken eine objektiv gegebene epistemische Nutzenfunktion für die möglichen Ausgänge jeder wissenschaftlichen Untersuchung ergäbe. Denn die verschiedenen epistemischen Werte sind jeder für sich nicht vollkommen präzise und können in vielen Fällen miteinander konfligieren, wie bereits Kuhn (1977, 322) feststellte. Ihre Anwendung auf eine konkrete wissenschaftliche Untersuchung macht ein individuelles Werturteil erforderlich.

Eine zusätzliche Komplikation ergibt sich daraus, dass epistemische und praktische Ziele wissenschaftlicher Untersuchungen typischerweise miteinander verflochten sind. Nehmen wir das Beispiel Galieo Galileis, der sich (wie er später im Sidereus Nuncius berichtete) im Jahre 1609 mit dem Phänomen der Lichtbrechung beschäftigte. Ein zentrales Anliegen dieser Bemühungen war die Verbesserung des Teleskops - sein Erfolg dabei ermöglichte ihm später ein einträgliches Geschäft mit der Herstellung von Teleskopen. Sicher war jedoch ein Ziel seiner Beschäftigung mit dem Teleskop auch, genauere Beobachtungen der Himmelsphänomene vornehmen zu können. Zählt also das Ziel der Verbesserung des Teleskops bei Galileis optischen Studien zu den epistemischen oder zu den nichtepistemischen Werteinflüssen? Eine instruktive Antwort auf diese Frage scheint es nicht zu geben. Die Ziel- und Wertvorstellungen, welche die Dynamik des wissenschaftlichen Erkenntnisprozesses vorantreiben, können nicht eindeutig in epistemische und nicht-epistemische, in inner- und außerwissenschaftliche Kategorien klassifiziert werden.

Es ergibt sich insgesamt, dass die Annahme, es gebe für jede Untersuchung eine bestimmte, richtige Verteilung der induktiven Risiken, nicht haltbar ist. Für ihre Veranschlagung und somit für methodologische Entscheidungen gibt es immer Spielraum. Individuell voneinander abweichende methodologische Entscheidungen, die unterschiedliche Aufteilungen der induktiven Risiken implizieren, kann man also nicht einfach als Abweichungen von einem Ideal der prozeduralen

3 Siehe Wilholt 2009, §6 für eine genauere Beschreibung dieser Möglichkeit. 
Objektivität analysieren, denn ein neutraler Standard für richtige Balance der induktiven Risiken existiert nicht.

\section{Die Perspektive der sozialen Erkenntnistheorie}

Dieses Ergebnis wäre für sich genommen sicher sehr unbefriedigend. Müssen wir uns achselzuckend mit dem Befund abgeben, dass wir in scheinbar offensichtlichen Fällen wie der Industrieforschung zur Wirkung geringer Dosen von Bisphenol A in Wirklichkeit gar keinen Mangel an prozeduraler Objektivität konstatieren können, weil nun einmal verschiedene Wissenschaftler die Verteilung der akzeptablen induktiven Risiken einer Untersuchung mit gleichem Recht auf verschiedene Weisen veranschlagen? Auf den folgenden Seiten skizziere ich eine negative Antwort auf diese Frage. Dem Grundgedanken der prozeduralen Objektivität lässt sich durchaus ein solider und aussagekräftiger Sinn geben, sobald man für seine Explikation die Ebene der wissenschaftlichen Gemeinschaft wählt.

Ein erster wichtiger Gesichtspunkt, der auf der Ebene der Forschungsgemeinschaften ins Blickfeld gerückt werden kann, betrifft deren konventionelle Standards (vgl. Wilholt 2009). Wissenschaftlerinnen und Wissenschaftler sind in der Praxis oft nicht frei in den methodologischen Entscheidungen, welche die Verteilung induktiver Risiken betreffen, sondern sie sind darin durch konventionelle Standards ihrer jeweiligen Community eingeschränkt. Ein augenfälliger solcher Standard ist die in vielen Disziplinen verbreitete Fünf-Prozent-Marke des maximal akzeptablen Signifikanzniveaus für statistische Untersuchungen. Dieser Standard trägt dazu bei, dass innerhalb und außerhalb der entsprechenden Forschergemeinschaften epistemische Akteure ein als ,statistisch signifikant' ausgewiesenes Ergebnis einzuschätzen wissen, ohne auf Mutmaßungen über etwaige individuelle Werturteile und entsprechende Verteilungen induktiver Risiken angewiesen zu sein. Dafür ist nicht entscheidend, dass die Marke bei $5 \%$ liegt, sondern dass alle dieselbe Marke anerkennen. Es handelt sich also bei einem solchen Standard um die Lösung eines Koordinationsproblems - mit anderen Worten, um eine Konvention. Für bestimmte Verfahrensweisen ist so eine Standardisierung möglich - und in diesem Sinne auch eine Objektivierung von Verfahren. Wegen des konventionellen Charakters solcher Standards sind sie aber nur als Errungenschaften der wissenschaftlichen Gemeinschaft verständlich.

Übrigens liegt auch im Bisphenol-A-Beispiel eine Verletzung konventioneller methodologischer Standards vor, weil die in Rede stehenden Studien das Erfordernis einer Positivkontrolle vernachlässigt haben. Durch die Verabreichung einer gut charakterisierten östrogen wirkenden Substanz an eine Positivkontrollgruppe wäre die mangelnde Eignung der Versuchstiere sichtbar geworden. Dies haben die meisten Industriestudien jedoch unterlassen; in zwei Fällen haben sie sogar Positivkontrollen durchgeführt und deren Ergebnisse später unterdrückt (vom Saal/Hughes 2005, 928-9; vom Saal/Welshons 2005, 52). Das Beispiel illustriert die Bandbreite konventioneller Standards, welche indirekt diejenigen 
methodologischen Entscheidungen einschränken, mittels derer sich die Verteilung der induktiven Risiken beeinflussen lässt.

Konventionelle Standards spielen insbesondere deshalb eine elementare Rolle für die soziale Erkenntnistheorie der Wissenschaften, weil sie die Ausbildung und Bewahrung von epistemischem Vertrauen in die Ergebnisse der Wissenschaften möglich machen. Dieses ist sowohl innerhalb der Wissenschaften für die arbeitsteilige Organisationsweise der Forschung unbedingt erforderlich, als auch eine Voraussetzung für die gesellschaftlichen Funktionen der Wissenschaft als Bereitsteller möglichst verlässlicher Informationen. Würde die Einschätzung der Verlässlichkeit eines Forschungsergebnisses jedes Mal eine Einsicht in die Struktur der Wertvorstellungen verlangen, die der Untersuchung zugrundegelegt wurden, wäre die Entwicklung von epistemischem Vertrauen praktisch unmöglich. Erst die impliziten Beschränkungen, die der Verteilung induktiver Risiken durch konventionelle Standards auferlegt werden, ermöglichen es Laien wie Experten, die Verlässlichkeit von als ,Ergebnis ${ }^{6}$ kommunizierten Behauptungen einschätzen zu lernen.

Man kann daher in konventionellen methodologischen Standards ein Element prozeduraler Objektivität und einen genuin sozialen Mechanismus der Verhinderung oder zumindest Einschränkung individueller Einseitigkeiten in der Wissenschaft sehen. Denn die Standards können zwar gegebenenfalls von einzelnen Individuen befolgt werden, ihre erkenntnistheoretischer Sinn ergibt sich jedoch erst aus ihrem konventionellen Charakter und ihrer Bedeutung für die Ausbildung und Bewahrung epistemischen Vertrauens. Ohne diesen sozialen Kontext wären sie nur willkürliche Setzungen ohne erkenntnistheoretische Relevanz.

Allerdings sollte es in Sachen prozeduraler Objektivität aus mindestens zwei Gründen nicht allein beim sozialen Mechanismus der konventionellen Standards bleiben. Erstens sind konventionelle Standards zwar offensichtlich geeignet, den Einfluss individueller Präferenzen auf Untersuchungsergebnisse einzudämmen, haben aber wenig Zugkraft gegen kollektiv geteilte Einseitigkeiten wie etwa allgemein verbreitete Vorurteile. Zweitens lassen sich sicher nicht alle methodologischen Entscheidungen mittels Konventionen standardisieren (sondern nur solche, die auf robuste Weise in geläufige Verfahren eingebettet sind).

Es gibt aber zum Glück ein weiteres genuin soziales Moment prozeduraler Objektivität in den Wissenschaften, das weit über den begrenzten Bereich standardisierter Verfahren hinausgreift. Dieses Moment besteht in der Pluralität verschiedener Ansätze und im Mechanismus wechselseitiger Kritik. Wissenschaftliche Gemeinschaften sind idealerweise sozial so organisiert, dass abweichende Ansichten und Ansätze ermöglicht und kritische Interaktionen gefördert werden. Individuell einseitige Werteinflüsse können sich ausgleichen und sogar produktiv stimulieren. Damit dieses Element der prozeduralen Objektivität (vgl. Fine 1998) zur Entfaltung kommt, müssen innerhalb der wissenschaftlichen Gemeinschaften soziale Strukturen vorliegen, die einen produktiven Pluralismus begünstigen. Helen Longino (1990, Kap. 4) hat vorgeschlagen, dass idealerweise unter anderem eine grundsätzliche Gleichheit der intellektuellen Autorität sowie das Vorliegen anerkannter Kanäle der Kritik zu diesen sozialen Voraussetzungen gehören. 
Die Begünstigung eines produktiven Pluralismus ist an manchen Punkten tatsächlich sehr tief in den sozialen Mechanismen der Wissenschaft verankert. Ein Beispiel dafür ist das Wirken der Prioritätsregel, derzufolge wissenschaftliche Anerkennung im Wesentlichen nur dem ersten Forscher, der ein bestimmtes Ergebnis liefert, zugesprochen wird. Die Aussichten darauf hängen deshalb für einen individuellen Forscher nicht nur vom intrinsischen Erfolgspotenzial des von ihm betriebenen Forschungsansatzes ab, sondern auch von der Gesamtzahl der gleichzeitig diesen Ansatz mit demselben Ziel verfolgenden Forscher. Ist diese Zahl zu groß, kann der individuelle Forscher wahrscheinlich seine Aussichten auf wissenschaftliche Anerkennung vergrößern, wenn er zu einem anderen, weniger überlaufenen Forschungsansatz wechselt - selbst wenn dieser ein geringeres intrinsisches Erfolgspotenzial hat. Auf diese Weise wird begünstigt, dass ein Teil des kollektiven Forschungsaufwandes auf Vorhaben verwandt wird, die aus Sicht der Mehrheit weniger als optimale Erfolgsaussichten besitzen (vgl. Kitcher 1993, Kap. 8).

Die Prioritätsregel ist nur ein Beispiel für ein Element der sozialen Organisation der Wissenschaften, das dazu beiträgt, dass Vielfalt und wechselseitige Kritik begünstigt werden. Zu entscheidenden sozialen Mechanismen gehören außerdem die offenen Kommunikationsformen der Wissenschaften sowie die Art und Weise, wie der Zugang zu Publikationsmöglichkeiten geregelt ist, und wie wissenschaftliche Anerkennung von Publikationserfolgen und individuelle Karrieren wiederum von wissenschaftlicher Anerkennung abhängen. Die prozedurale Objektivität der Wissenschaften hängt ganz wesentlich davon ab, dass diese sozialen Organisationsformen so gestaltet sind, dass die Pluralität von Ansätzen, die wechselseitige Kritik und natürlich auch die produktive Aufnahme von Kritik begünstigt wird.

Das Beispiel der Tierversuche zur Wirkung geringer Dosen von Bisphenol A verdeutlicht auch diese Dimension prozeduraler Objektivität. Denn die toxikologische Community hat eben nicht nur Studien mit dem problematischen Versuchstierstamm hervorgebracht, sondern auch eine ganze Reihe weiterer, hauptsächlich öffentlich geförderter Studien mit ganz anderen Ergebnissen, und schließlich, in Gestalt der Anstrengungen vom Saals und seiner Kollegen, auch vergleichende Untersuchungen dieser unterschiedlichen Studien, bei denen die Ursachen der abweichenden Ergebnisse und das Problem mit den Östrogen-unempfindlichen Versuchstieren identifiziert werden konnten.

Die prozedurale Objektivität hat hier im Ergebnis durchaus etwas mit der Balance der induktiven Risiken zu tun. Nur manifestiert sie sich eben nicht darin, dass alle einer verbindlichen Aufteilung der Risiken möglichst nahe kommen, sondern darin, dass durch eine Bandbreite verschiedener Experimente mit abweichenden methodologischen Entscheidungen unterschiedliche Aufteilungen der induktiven Risiken realisiert werden und so die Gefahr der Einseitigkeit gebannt wird. Es ist eine für das Verständnis der prozeduralen Objektivität kritische Einsicht, dass der analytischen Kraft einer Verbindung von Pluralismus und Gleichgewicht induktiver Risiken Grenzen gesetzt sind. So versucht zwar Miriam Solomon (2001, insb. Kap. 8), den Wert von Pluralismus und kritischer Interaktion in den Wissenschaften darin auszumachen, dass sie idealerweise dazu 
führt, dass sich alle individuellen Einseitigkeiten, in ihrer eigenen Terminologie „non-empirical decision vectors“, letztlich zu Null addieren. Im Lichte der im vorigen Abschnitt dargestellten Argumente gegen die Vorstellung einer einzigen, objektiv korrekten oder idealen Verteilung der induktiven Risiken erscheint eine konsequentialistische Konzeption prozeduraler Objektivität in diesem Sinne jedoch hochproblematisch. Sie würde auf der Ebene der sozialen Erkenntnistheorie den Versuch wiederholen, an der schon eine individualistische Objektivitätskonzeption scheitern musste. Eine Alternative dazu ist es, den Beitrag von Pluralismus und wechselseitiger Kritik zur Objektivität genuin prozedural zu verstehen: Das hieße, den Praktiken einer wissenschaftlichen Gemeinschaft in diesem Sinne das Vorliegen von Objektivität zuzuschreiben, wenn sie sich nach den geeigneten (Pluralismus und wechselseitige Kritik fördernden) Verfahren richtet unabhängig davon, welche Verteilung von Einflussfaktoren im Einzelfall daraus resultiert. ${ }^{4}$

Das Beispiel der Forschung zu Bisphenol A verdeutlicht aber noch einen weiteren wichtigen Gesichtspunkt: Wir dürfen nicht als gegeben voraussetzen, dass gute Bedingungen für Vielfalt und wechselseitige Kritik stets durch eine vom Streben nach Anerkennung, einer Prioritätsregel und vergleichbaren Mechanismen geführte unsichtbare Hand automatisch hergestellt werden. In diesem wie in vielen anderen Fällen hängt prozedurale Objektivität daran, dass die öffentliche Hand Mittel für ergebnisoffene Forschung einsetzt - gerade dort, wo mächtige private Interessen im Spiel sind.

\section{Lohnende Herausforderungen}

Auch wenn die sozialepistemologische Sichtweise verspricht, Kriterien prozeduraler Objektivität zu identifizieren, wirft sie offenbar zugleich viele neue Fragen auf: Wie genau ist das Verhältnis der beiden hier skizzierten Elemente (konventionelle Standards und Pluralismus) zu verstehen? Bieten konsequentialistische oder genuin prozedurale Kriterien die überzeugendste Analyse des in Rede stehenden Objektivitätsbegriffs? Kann die Betrachtung ganz auf der Ebene wissenschaftlicher Gemeinschaften bleiben, oder muss der weitere gesellschaftliche Kontext, wie etwa der Einfluss wirtschaftlicher Interessen, mit in die Analyse eingehen?

Der hier von mir skizzierte Sinn von Objektivität setzt also der philosophischen Analyse noch manchen Widerstand entgegen. Lohnt er überhaupt den großen Aufwand und die Aufmerksamkeit der Wissenschaftsphilosophie? Ein kurzes Plädoyer für eine positive Antwort auf diese Frage möchte ich mir abschließend erlauben. Die Wissenschaftsphilosophie war lange Zeit stark von Interessen geprägt, die man im weitesten Sinne als naturphilosophische bezeichnen könnte - also in etwa durch ein Interesse daran, was eigentlich die Ergebnisse naturwissenschaftlicher Forschung an grundsätzlichen Einsichten über unsere Welt

\footnotetext{
4 Solomon stellt ihre Ideen nicht ausdrücklich als Explikation eines Objektivitätsbegriffs vor. Für ein genuin prozedurales Verständnis der Objektivität argumentiert ausführlich Fine 1998; als Ausformulierung eines solchen lassen sich meines Erachtens Longinos 1990; 2002 Auffassungen verstehen.
} 
und unsere Stellung in ihr bereithalten. Dadurch bedingt, liegt ein traditioneller Interessenschwerpunkt der Wissenschaftsphilosophie auf der naturwissenschaftlichen Grundlagenforschung. Der Einfluss einseitiger Interessen auf Forschungsergebnisse ist in der Grundlagenforschung vermutlich geringer als bei anwendungsorientierter Wissenschaft. ${ }^{5}$ Die prozedurale Objektivität ist hier deshalb möglicherweise kein sehr augenfälliges Problemfeld; dagegen scheinen Fragen nach der objektiven Wahrheit in demjenigen Sinne, um den es in der eingangs angesprochenen Realismusdebatte geht, hier eher beheimatet zu sein - etwa die Frage, ob uns die Stringtheorie wirklich über eine Welt von Gegenständen unterrichtet, die unabhängig von unserem Nachdenken über sie existieren.

Dass die Wissenschaftsphilosophie ihren Fokus stärker auch auf anwendungsorientierte Forschung ausrichten sollte, folgt meines Erachtens nicht in erster Linie daraus, dass Grundlagenforschung heute den deutlich geringeren Teil aller Forschungsaktivitäten ausmacht. ${ }^{6}$ Entscheidender ist, dass gerade anwendungsorientierte naturwissenschaftliche Forschung immer bestimmender für viele Fragen wird, die in unserer Lebenswelt Relevanz haben, wie etwa die Frage, welche Auswirkungen Bisphenol A auf unsere Gesundheit hat, welche Therapie die besten Heilungschancen für eine bestimmte Krankheit bietet, welche Gefahren die gentechnische Manipulation von Nutzpflanzen birgt, ob von Endlagerstätten für nukleare Abfälle die Gefahr einer radioaktiven Verseuchung des Grundwassers ausgeht, ob der Klimawandel anthropogen ist und durch welche Maßnahmen wir ihn beeinflussen können. Bei diesen und vielen anderen Themen ist die Frage, ob und in welchem Sinne wir uns auf eine prozedurale Objektivität der einschlägigen wissenschaftlichen Forschungen verlassen können, von vitalem Interesse. Sich auf den Standpunkt des Wissenschaftsskeptizismus zurückzuziehen und zu konstatieren, der Begriff der Objektivität sei lediglich ein Instrument der Geltungsrhetorik des wissenschaftlichen Standes, worauf der Mainstream der Science and Technology Studies lange Zeit hinauslief, löst das Problem nicht. Denn da die Schwierigkeit erst dadurch eigentlich entsteht, dass uns als Bürgern, Konsumenten und Patienten das nicht immer gleich gute Funktionieren von Wissenschaft als Quelle verlässlichen Wissens nicht gleichgültig sein kann, kann eine ironische Einstellung zu den Wissensansprüchen der Wissenschaften nicht als Antwort auf die dadurch gestellte Herausforderung gelten. ${ }^{7}$ Für die Wissenschaftsphilosophie

${ }^{5}$ Ein Grund dafür ist darin zu sehen, dass die mit den induktiven Risiken verbundenen praktischen Risiken bei manchen anwendungsorientierten Forschungsfeldern von verschiedenen Parteien getragen werden. Bei der Prüfung der Schädlichkeit eines Lebensmittelzusatzes beispielsweise tragen die Konsumenten das Risiko eines falsch-negativen Ergebnisses, die Hersteller dasjenige eines falsch-positiven. In Fällen, in denen keine im Vorhinein erkennbare Verteilung der zwei Risikotypen auf verschiedene Akteure besteht, ist das Auftreten von Einseitigkeiten aufgrund von Interessenkonflikten nicht wahrscheinlich. Vgl. Wilholt 2006, 70-1.

6 Insgesamt wurden beispielsweise in den USA im Jahr 2007 aus staatlichen und privaten Quellen für Grundlagenforschung 64,4 Mrd. \$ ausgegeben, für angewandte Forschung 81,2 Mrd. \$ und für Entwicklung 222,5 Mrd. \$ - laut Statistik der National Science Foundation (siehe http://www.nsf.gov/statistics/ und http://www.aaas.org/spp/rd/totalr07t.pdf), wobei Grundlagenforschung bei der Erhebung bereits sehr großzügig definiert wird als „research for which the primary aim of the investigator is a fuller knowledge or understanding of the subject under study rather than a specific application thereof" (Committee on National Statistics 2004, $31-2)$.

7 Bemerkenswerterweise zeichnet sich die Möglichkeit einer Trendwende der Science and 
besteht deshalb in der Artikulation eines kohärenten und realistischen Begriffs der Objektivität wissenschaftlicher Vorgehensweisen eine wichtige Aufgabe - und diese muss sich wesentlich auf die sozialen Charakteristika wissenschaftlicher Forschung stützen.

\section{Bibliographie}

Churchman, C. W. (1948), Theory of Experimental Inference, New York

Collins, H. M. (1985), Changing Order: Replication and Induction in Scientific Practice, London

- /R. Evans (2002), The Third Wave of Science Studies: Studies of Expertise and Experience, in: Social Studies of Science 32, 235-296

Committee on National Statistics (2004), Measuring Research and Development Expenditures in the U.S. Economy, Washington

Douglas, H. (2000), Inductive Risk and Values in Science, in: Philosophy of Science $67,559-579$

- (2004), The Irreducible Complexity of Objectivity, in: Synthese 138, 453-473

Fine, A. (1998), The Viewpoint of No-One in Particular, in: Proceedings and Addresses of the American Philosophical Association 72, 9-20

Goldman, A. I. (1999), Knowledge in a Social World, Oxford

Hacking, I. (1999), The Social Construction of What?, Cambridge/MA

Hempel, C. G. (1965), Science and Human Values, in: Aspects of Scientifc Explanation, New York, 81-96

Kitcher, P. (1993), The Advancement of Science: Science without Legend, Objectivity without Illusions, New York

- (2001), Science, Truth, and Democracy, New York

Kourany, J. A. (2003), A Philosophy of Science for the Twenty-First Century, in: Philosophy of Science 70, 1-14

Kuhn, T. S. (1962), The Structure of Scientific Revolutions, Chicago.

- (1977), Objectivity, Value Judgment, and Theory Choice, in: The Essential Tension, Chicago, 320-339

Latour, B./S. Woolgar (1979), Laboratory Life: The Construction of Scientific Facts, Beverly Hills

Longino, H. E. (1990), Science as Social Knowledge: Values and Objectivity in Scientific Inquiry, Princeton

- (1996), Cognitive and Non-Cognitive Values in Science: Rethinking the Dichotomy, in: L. H. Nelson/J. Nelson (Hrsg.), Feminism, Science, and the Philosophy of Science, Dordrecht, 39-58

- (2002), The Fate of Knowledge, Princeton

McMullin, E. (1983), Values in Science, in: P. D. Asquith/T. Nickles (Hrsg.), PSA 1982, Bd. 2, East Lansing, 3-28

Megill, A. (1994), Introduction: Four Senses of Objectivity, in: A. Megill (Hrsg.), Rethinking Objectivity, Durham, 1-20

Rudner, R. (1953), The Scientist qua Scientist Makes Value Judgments, in: Philosophy of Science 20, 1-6

Technology Studies ab, die aufgrund genau dieser Erfordernisse darauf hinausliefe, wissenschaftliche Normen und Standards wieder ernster zu nehmen. S. insb. Collins/Evans 2002. 
Vom Saal, F. S./C. Hughes (2005), An Extensive New Literature Concerning LowDose Effects of Bisphenol A Shows the Need for a New Risk Assessment, in: Environmental Health Perspectives 113, 926-933

- /W. V. Welshons (2005), Large Effects from Small Exposures. II. The Importance of Positive Controls in Low-Dose Research on Bisphenol A, in: Environmental Research 100, 50-76

Solomon, M. (2001), Social Empiricism, Cambridge/MA

Wilholt, T. (2006), Design Rules: Industrial Research and Epistemic Merit, in: Philosophy of Science 73(1), 66-89

- (2009), Bias and Values in Scientific Research, in: Studies in History and Philosophy of Science 40, 92-101 